\section{OPEN ACCESS}

Edited by:

Jorge Blanco,

Universidade de Santiago

de Compostela, Spain

Reviewed by:

Patrick Fach

Agence Nationale de Sécurité Sanitaire de l'Alimentation, de

l'Environnement et du Travail,

(ANSES), France

Azucena Mora Gutiérrez,

Universidade de Santiago

de Compostela, Spain

*Correspondence:

Ken-ichi Lee

leek@niid.go.jp

Specialty section: This article was submitted to

Infectious Diseases,

a section of the journal

Frontiers in Microbiology

Received: 03 February 2017

Accepted: 05 April 2017

Published: 20 April 2017

Citation:

Lee K, Morita-Ishihara T, lyoda S, Ogura Y, Hayashi T, Sekizuka T

Kuroda M, Ohnishi M and EHEC Working

Group in Japan (2017)

A Geographically Widespread

Outbreak Investigation

and Development of a Rapid

Screening Method Using Whole

Genome Sequences

of Enterohemorrhagic Escherichia coll

O121. Front. Microbiol. 8:701.

doi: 10.3389/fmicb.2017.00701

\title{
A Geographically Widespread Outbreak Investigation and Development of a Rapid Screening Method Using Whole Genome Sequences of Enterohemorrhagic Escherichia coli 0121
}

Ken-ichi Lee ${ }^{1 *}$, Tomoko Morita-Ishihara', Sunao lyoda', Yoshitoshi Ogura', Tetsuya Hayashi², Tsuyoshi Sekizuka ${ }^{3}$, Makoto Kuroda ${ }^{3}$, Makoto Ohnishi ${ }^{1}$ and EHEC Working Group in Japan ${ }^{4}$

\begin{abstract}
'Department of Bacteriology I, National Institute of Infectious Diseases, Tokyo, Japan, ${ }^{2}$ Department of Bacteriology, Faculty of Medical Sciences, Kyushu University, Fukuoka, Japan, ${ }^{3}$ Laboratory of Bacterial Genomics, Pathogen Genomics Center, National Institute of Infectious Diseases, Tokyo, Japan, ${ }^{4}$ Local Public Health Institutes, Japan
\end{abstract}

From 2014 to 2015, we investigated a suspected nationwide outbreak of enterohemorrhagic Escherichia coli serogroup 0121. However, similar pulsed field gel electrophoresis (PFGE) profiles and the lack of epidemiological links between the isolates made detection of the outbreak difficult. To elucidate a more precise genetic distance among the isolates, whole genome sequence (WGS) analyses were implemented in the investigation. The WGS-based single nucleotide polymorphism (SNP) analysis showed that 23 out of 44 isolates formed a distinct cluster (the number of intra-cluster SNPs was $\leq 8)$. Specific genomic regions in the clustered isolates were used to develop a specific PCR analysis. The PCR analysis detected all the clustered isolates and was suitable for rapid screening during the outbreak investigation. Our results showed that WGS analyses were useful for the detection of a geographically widespread outbreak, especially for isolates showing similar PFGE profiles and for the development of a rapid and cost-effective screening method.

Keywords: molecular typing, diarrhea, phylogeny enterohemorrhagic Escherichia coli, disease outbreak, single nucleotide polymorphism

\section{INTRODUCTION}

Enterohemorrhagic Escherichia coli (EHEC) is a leading cause of foodborne illness worldwide that causes diarrhea, hemorrhagic colitis, and life-threatening hemolytic uremic syndrome (Pennington, 2010). More than 3,000 cases of infection are reported annually in Japan (Infectious Agents Surveillance Report, 2016), and the isolates are extensively monitored to detect nationwide outbreaks. All culture-positive cases of EHEC infection are reported to the National Institute of Infectious Diseases, Japan, irrespective of the serogroup. Currently, national surveillance of EHEC is performed using multilocus variable-number tandem-repeat analysis (MLVA) for serogroups O157, O26, and O111 (Izumiya et al., 2010) and pulsed field gel electrophoresis (PFGE) for the other serogroups. 
Pulsed field gel electrophoresis can be used for the molecular typing of all EHEC serogroups and is available in many public health laboratories. Therefore, PFGE is regarded as the "gold standard" for the molecular typing of EHEC. However, PFGE has several drawbacks. The band recognition can be subjective, and a different threshold for a band position tolerance can generate different results. Additionally, fragments with the same size do not always have similar sequences and may not reflect the phylogeny (Noller et al., 2003; Li et al., 2009). Recently, molecular typing using whole genome sequence (WGS) analysis has become available. Phylogenetic analyses using single nucleotide polymorphisms (SNPs) or k-mer based methods from WGSs provide higher resolution for typing than the conventional typing methods. WGS typing has been widely used for various purposes, including EHEC outbreak surveillance (Grad et al., 2012), investigations of nosocomial infections of methicillin-resistant Staphylococcus aureus (Harris et al., 2013), and long-term tracing of Clostridium difficile in a community (Eyre et al., 2013). WGS typing is useful especially in outbreak investigations in which the isolates are highly clonal and difficult to distinguish using conventional methods (Salipante et al., 2015; Bekal et al., 2016). A recently developed network sharing WGS database has enabled more effective tracing of foodborne pathogens at the national and international levels, such as Genome Trakr Network (Allard et al., 2016) and EnteroBase ${ }^{1}$. Moreover, gene information specific to an outbreak strain can be extracted simultaneously (Grad et al., 2012; Sekizuka et al., 2015).

EHEC O121 is one of the most common non-O157 serogroup. In Japan, 12-96 isolates were reported annually during the past decade, which correspond to $0.6-4.4 \%$ of all EHEC cases ${ }^{2}$. We detected several EHEC O121 isolates showing high PFGE similarity in the national surveillance program from 2014 to 2015. Because most of the isolates did not have epidemiological links with other isolates and because PFGE did not have sufficient discriminatory power for the isolates, WGS typing was used to identify links between the isolates. Furthermore, we developed a rapid and cost-effective screening method for many samples to detect outbreak strains using WGS information.

\section{MATERIALS AND METHODS}

\section{EHEC 0121 Isolates Used in This Study}

From June 2014 to July 2015, EHEC O121 isolates that showed similar PFGE profiles were identified from the national EHEC surveillance. Personal information of the patients was completely anonymized and only the information of isolation date, isolation site and symptoms was used in this study. All but one isolate (141341, isolated from an asymptomatic carrier) were isolated from patients that showed abdominal pain, diarrhea or hemolytic uremic syndrome and their family. Five isolates (150337-150341) were obtained from the same family. However, there was no clear epidemiological link in the other isolates. Forty-one isolates

${ }^{1}$ http://enterobase.warwick.ac.uk/

${ }^{2}$ http://www.nih.go.jp/niid/en/iasr-vol37-e/865-iasr/6488-435te.html
(140961-151387) that formed a cluster with more than 85\% PFGE similarity were used for the WGS analyses (Table 1). Additionally, three previously collected isolates (121512, 132137, and 140452) were used. The serial isolate numbers provided in Table 1 were used throughout this study. The genes for Shiga toxin $(s t x)$ and their subtypes were determined by PCR (Scheutz et al., 2012).

\section{PFGE and MLST Analyses}

Pulsed field gel electrophoresis was performed as described elsewhere (Pei et al., 2008), with minor modifications. In brief, bacterial cells on an agar medium were suspended in $200 \mu \mathrm{l}$ of distilled water, and the samples were mixed with an equal amount of $1 \%$ SeaKem Gold agarose (Lonza, Basel, Switzerland) to induce plug formation. After appropriate preparations for restriction endonuclease digestion, the DNA in each plug was digested with $30 \mathrm{U}$ of XbaI (Roche Diagnostics, Basel, Switzerland) at $37^{\circ} \mathrm{C}$ for $2.5 \mathrm{~h}$. The PFGE was performed using a $\mathrm{CHEF}$ DRIII system (Bio-Rad Laboratories, Hercules, CA, USA) with the following run parameters: a switch time of 2.2-54.2 and a run time of $21 \mathrm{~h}$. Salmonella enterica serovar Braenderup H9812 was used as the size standard. Analysis in BioNumerics 6.6 (Applied Math, Kortrijk, Belgium) was performed with a $1 \%$ position tolerance. An unweighted pair group method with arithmetic mean (UPGMA) clustering algorithm was used to create a hierarchical dendrogram, and the genetic similarity between the isolates was calculated using the Dice coefficient.

\section{WGS Analyses for the Phylogenetic Tree and Pairwise SNP Distances}

Whole genome sequences were obtained using MiSeq (Illumina, San Diego, CA, USA). The genomic DNA libraries were prepared using a Nextera XT DNA sample prep kit (Illumina). The pooled libraries were subjected to multiplexed paired-end sequencing $(300$ mer $\times 2)$. The sequence reads were assembled using the A5-miseq pipeline (Coil et al., 2015). The contig sequences were aligned with the contigs of isolate 1 using MUMmer version 3.2259 (Kurtz et al., 2004) to identify the conserved backbone (core genome) of these strains and the SNP sites. A 4,645,249 bp sequence was conserved in all the strains examined, with $>99 \%$ sequence identity and a $>2,000$ bp alignment length. The recombinogenic regions were removed by RecHMM (Zhou et al., 2014). Phylogenetic relationships were determined by reconstructing a phylogenetic tree using the maximum likelihood method based on the Tamura-Nei model with 1,000 bootstraps (Tamura and Nei, 1993) using the MEGA 7 software (Kumar et al., 2016). For the clustered isolates, a median joining network tree was constructed using the PopART software ver. $1.7^{3}$. The phylogenetic tree was also visualized with a partial national map of Japan using GenGIS software version 2.5.1 (Parks et al., 2013). In the map, each isolate was plotted on the prefectural capital of the isolation site. To confirm that the clustered isolates were genetically distinct from the other isolates, a root-to-tip analysis was performed. The root-to-tip distance

${ }^{3}$ http://popart.otago.ac.nz 
TABLE 1 | Strain information used in this study.

\begin{tabular}{|c|c|c|c|c|c|}
\hline ID & Serial no. in this study & Isolation date & Prefecture & stx subtype & MLST \\
\hline 121512 & 1 & $2008-07-24$ & Okayama & $2 a$ & 655 \\
\hline 132137 & 2 & 2013-09-27 & Shiga & $2 a$ & 655 \\
\hline 140452 & 3 & 2014-03-13 & Niigata & $2 a$ & 655 \\
\hline 140961 & 4 & 2014-06-28 & Gumma & $2 a$ & 655 \\
\hline 140990 & 5 & 2014-06-25 & Ishikawa & $2 a$ & 655 \\
\hline 141004 & 6 & 2014-06-30 & Chiba & $2 a$ & 5536 \\
\hline 141202 & 7 & $2014^{a}$ & Shizuoka & $2 a$ & 655 \\
\hline 141247 & 8 & 2014-07-30 & Osaka & $2 a$ & 5536 \\
\hline 141341 & 9 & 2014-07-09 & Hiroshima & $2 a$ & 655 \\
\hline 141544 & 10 & $2014-07-24$ & Gumma & $2 a$ & 655 \\
\hline 142136 & 11 & 2014-08-29 & Miyagi & $1 a, 2 a$ & 655 \\
\hline 142321 & 12 & 2014-08-27 & Kagoshima & $2 a$ & 655 \\
\hline 142478 & 13 & 2014-10-09 & Yamagata & $2 a$ & 655 \\
\hline 142676 & 14 & 2014-12-01 & Osaka & $2 a$ & 655 \\
\hline 142774 & 15 & 2014-12-04 & Fukuoka & $2 a$ & 655 \\
\hline 150151 & 16 & 2014-12-03 & Ibaraki & $2 a$ & 655 \\
\hline 150152 & 17 & $2015-01-13$ & Ibaraki & $2 a$ & 655 \\
\hline 150174 & 18 & $2014-08-25$ & Fukuoka & $2 a$ & 655 \\
\hline 150213 & 19 & $2014-12-11$ & Shizuoka & $2 a$ & 655 \\
\hline 150238 & 20 & 2014-12-07 & Miyagi & $2 a$ & 655 \\
\hline 150239 & 21 & $2014-12-17$ & Miyagi & $2 a$ & 655 \\
\hline 150240 & 22 & $2014-12-22$ & Miyagi & $2 a$ & 655 \\
\hline 150241 & 23 & 2014-12-19 & Miyagi & $2 a$ & 655 \\
\hline 150242 & 24 & 2014-12-19 & Osaka & $2 a$ & 655 \\
\hline 150243 & 25 & $2014-12-24$ & Osaka & $2 a$ & 655 \\
\hline 150281 & 26 & $2014-12-15$ & Nara & $2 a$ & 655 \\
\hline 150337 & 27 & 2015-01-28 & Nagano & $2 a$ & 655 \\
\hline 150338 & 28 & 2015-01-28 & Nagano & $2 a$ & 655 \\
\hline 150339 & 29 & 2015-01-31 & Nagano & $2 a$ & 655 \\
\hline 150340 & 30 & 2015-01-31 & Nagano & $2 a$ & 655 \\
\hline 150341 & 31 & 2015-02-01 & Nagano & $2 a$ & 655 \\
\hline 150342 & 32 & 2014-06-30 & Kanagawa & $2 a$ & 655 \\
\hline 150373 & 33 & 2014-12-19 & Yamagata & $2 a$ & 655 \\
\hline 150375 & 34 & $2014^{a}$ & Tokyo & $2 a$ & 655 \\
\hline 150376 & 35 & $2014^{a}$ & Tokyo & $2 a$ & 655 \\
\hline 150387 & 36 & $2014-12-24$ & Aomori & $2 a$ & 655 \\
\hline 150393 & 37 & $2014^{a}$ & Shizuoka & $2 a$ & 655 \\
\hline 150395 & 38 & $2015^{a}$ & Hyogo & $2 a$ & 655 \\
\hline 150400 & 39 & 2014-12-10 & Miyazaki & $2 a$ & 655 \\
\hline 150542 & 40 & $2014-12-26$ & Okayama & $2 a$ & 655 \\
\hline 150616 & 41 & 2015-03-31 & Yamagata & $2 a$ & 655 \\
\hline 150977 & 42 & $2015-05-21$ & Kagawa & $2 a$ & 655 \\
\hline 151171 & 43 & 2015-06-17 & Kanagawa & $2 a$ & 655 \\
\hline 151387 & 44 & 2015-07-21 & Kanagawa & $2 a$ & 655 \\
\hline
\end{tabular}

a/solation date is not available.

of the clustered isolates and the most closely related isolate 18 were calculated using the Path-O-Gen software (Rambaut et al., 2016).

\section{In Silico Analyses of the Draft Genomes}

Three molecular typing schemes were applied to the contigs assembled as described above. Multilocus sequence typing
(MLST) was performed according to the protocols available in the E. coli MLST database ${ }^{4}$ (Wirth et al., 2006). Inc type of the plasmids were investigated using PlasmidFinder $1.3^{5}$.

${ }^{4}$ http://mlst.warwick.ac.uk/mlst/dbs/Ecoli

${ }^{5}$ https://cge.cbs.dtu.dk/services/PlasmidFinder/ 


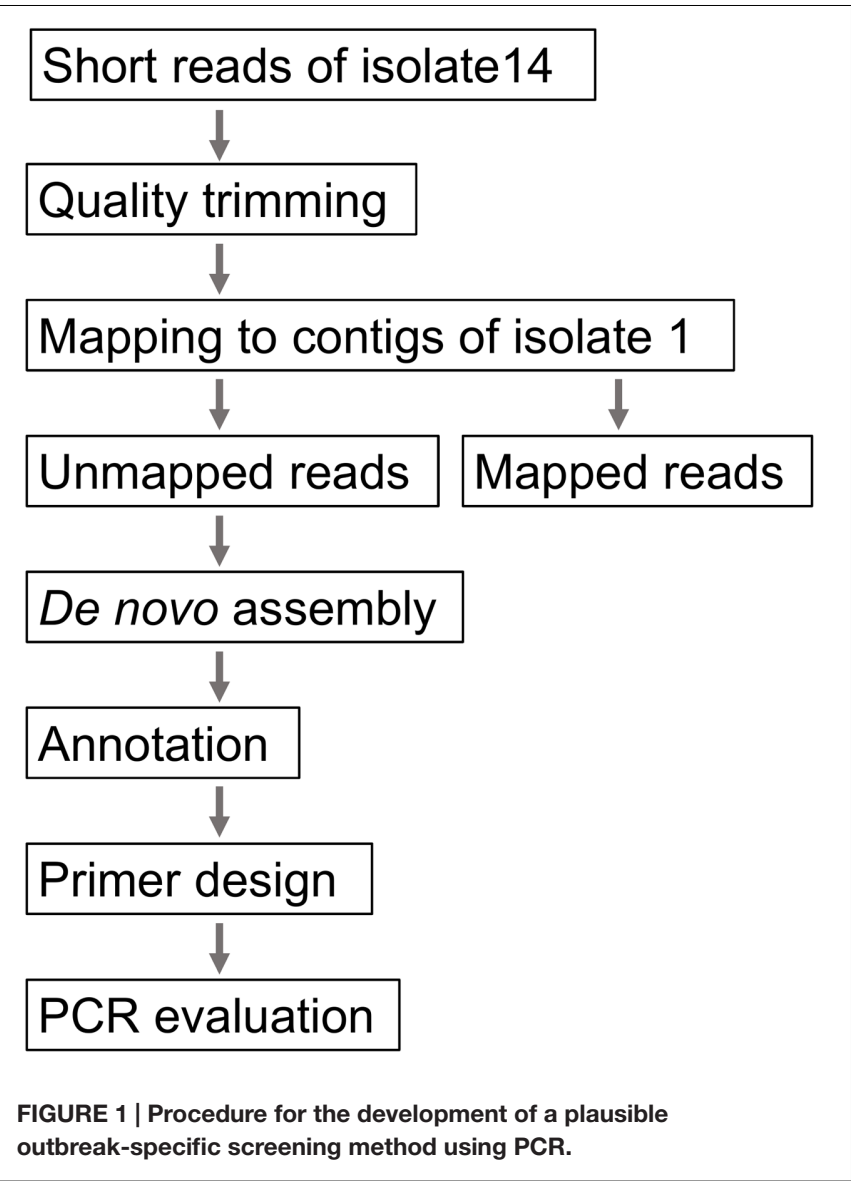

\section{Development of a Plausible Outbreak-specific Screening Method Using PCR}

The WGS analyses identified a cluster consisting of 23 isolates. We regarded this cluster as a plausible outbreak cluster. A rapid screening method using a PCR assay was developed in preparation for future outbreaks by the isolate and to trace the contaminated food product (Figure 1). First, short reads of one outbreak-associated isolate (isolate 14) were mapped to the contigs of isolate 1 using the CLC genomics workbench (QIAGEN, Inc., Valencia, CA, USA). Second, the unmapped reads were subjected to de novo assembly, and the generated contigs were annotated using the Microbial Genome Annotation Pipeline $\left(\mathrm{MiGAP}^{6}\right)$ annotation server. Third, two pairs of primers [HP-1 (5'-CGTTTGGCATACTGGGTTGC-3') and HP-2 (5'GTCTGACCAGAGCTCGCTTT-3'), which generate a 288 bp amplicon, and HP-5 (5' ${ }^{\prime}$ TTTACATGGCGGGGAATCGT- $\left.3^{\prime}\right)$ and HP-6 (5'-CCTGCACCCACCGTTCATAA- $\left.{ }^{\prime}\right)$, which generate a 609 bp amplicon] were constructed from the specific regions. The specificity and sensitivity were evaluated using all the O121 isolates described above and $11 \mathrm{EHEC}$ isolates belonging to other serogroups (O157, 1 isolate; O26, 10 isolates; O103, 4 isolates; O145, 4 isolates; and O165, 4 isolates).

\section{Accession Number}

The FASTQ sequences and assembled contigs used in this study were deposited in the DNA Data Bank of Japan ${ }^{7}$ under accession number DRA005295.

\section{RESULTS}

\section{PFGE, MLST and Plasmid Replicon Typing}

According to PFGE results, two major clusters were generated at the $99 \%$ threshold of the Dice similarity coefficient (Figure 2). The first cluster consisted of 22 isolates. Although these isolates were isolated over a 1-year period, the isolation sites of some of the isolates were separated by a great distance $(>1,000 \mathrm{~km})$. The other major cluster consisted of five isolates that were isolated from a single family. However, 36 isolates, including the two clusters described above, formed one cluster at the 95\% threshold. These isolates showing similar PFGE profiles were isolated over a 14-month period from a widespread area in Japan. Because no common epidemiologic factors linked these isolates, whether these isolates were derived from the same source was unclear. MLST did not provide sufficient resolution. All but two isolates belonged to ST 655. The other two isolates belonged to ST 5536, which had a one locus difference from ST 655 (Table 1). Plasmid replicon typing also did not have sufficient discriminatory power (Figure 1).

\section{Phylogenetic Analysis Using Whole Genome Sequences}

To gain a more precise insight into a set of isolates with similar PFGE profiles, WGSs were obtained and SNPs were extracted from the core genome of the 44 isolates used in this study. The concatenated alignment of 713 SNP sites located in the conserved backbone was used for further analyses. The maximum likelihood tree indicated that 23 isolates formed a cluster (Figure 3A). In the cluster, the pairwise SNP distances ranged from zero to eight, with mean and median values of 2.6 and 2.0, respectively (Figure 3B). The cluster could be subdivided into two groups consisting of 14 (upper part of Figure 3B) and 9 (lower part of Figure 3B) isolates. The geographical location and date information for the isolates did not distinguish these two groups (Figure 4). One of the groups included isolates from the same family members, in which the maximum pairwise SNP distance was five. The most closely related isolate to the cluster was isolate 18 (the mean pairwise SNP was 17). The other isolates had 55 or more pairwise SNPs from the cluster. The root-to-tip analysis reinforced that the clustered isolates were genetically distinct from the other isolates (Figure 5). This analysis shows a proportionate relationship between isolation interval (x-axis) and distance from the root of the phylogenetic tree (y-axis) if the isolates were originated from the same ancestor, assuming a uniform mutation rate. Therefore,

${ }^{7}$ http://www.ddbj.nig.ac.jp

${ }^{6}$ http://www.migap.org/index.php/en 


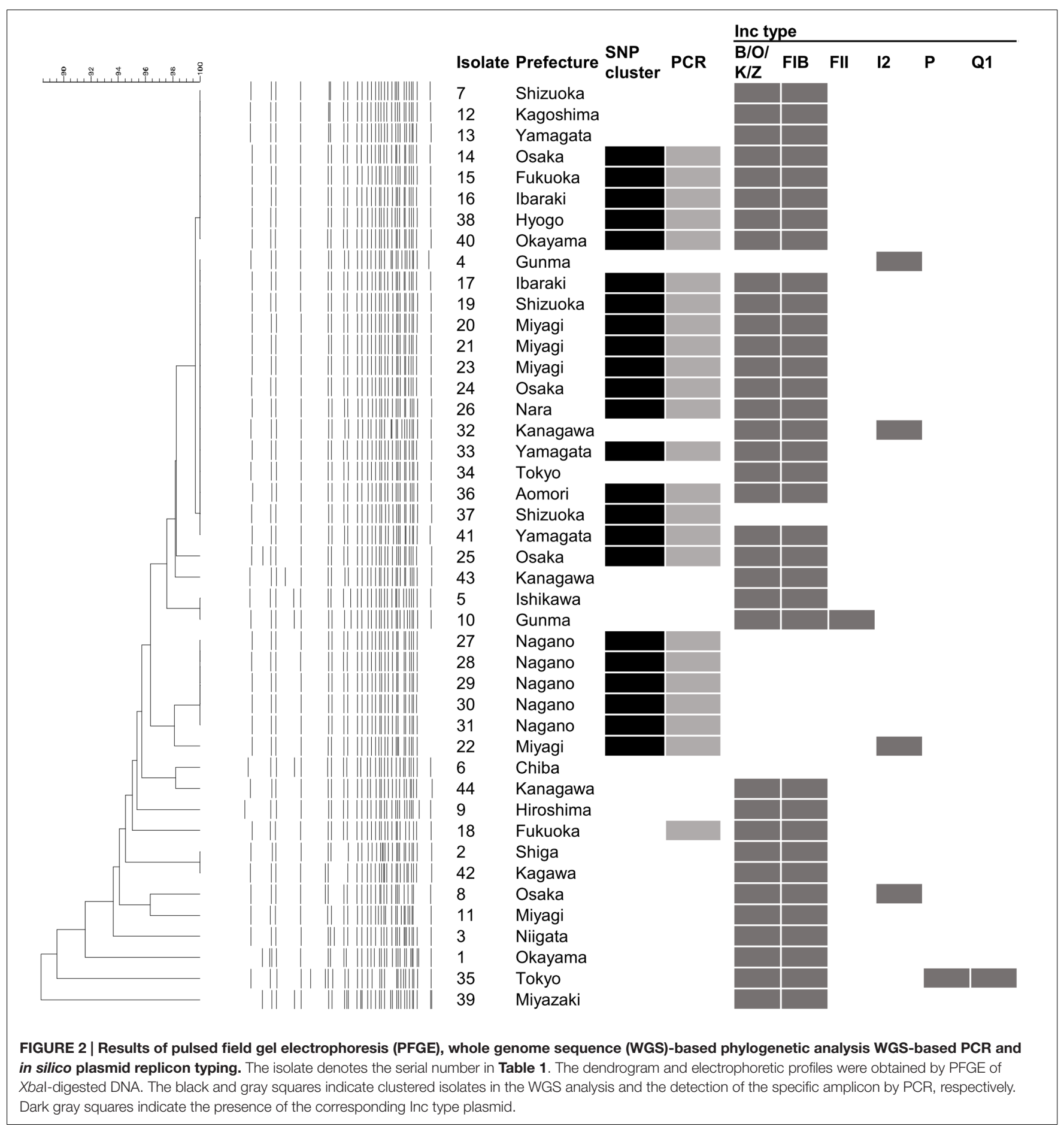

the isolates from the same ancestor will be plotted along a line. Isolates 18 and 41 were the outliers from the clustered isolates. The phylogenetic analysis results suggested that the clustered isolates and isolate 18 had different ancestors. However, isolate 41 was included in the cluster by the phylogenetic analysis, which suggested that bacterial multiplication might be inhibited in certain environments, such as food stored in low temperature.

\section{Development of a Plausible Outbreak-specific Screening Method Using PCR}

To perform rapid and more precise screening for the clustered isolates, a WGS-based PCR method was developed. When short reads of isolate 14 were mapped to the contigs of isolate $1,1.05 \%$ of the short reads $(22,962 / 2,177,097$ reads; average 
A

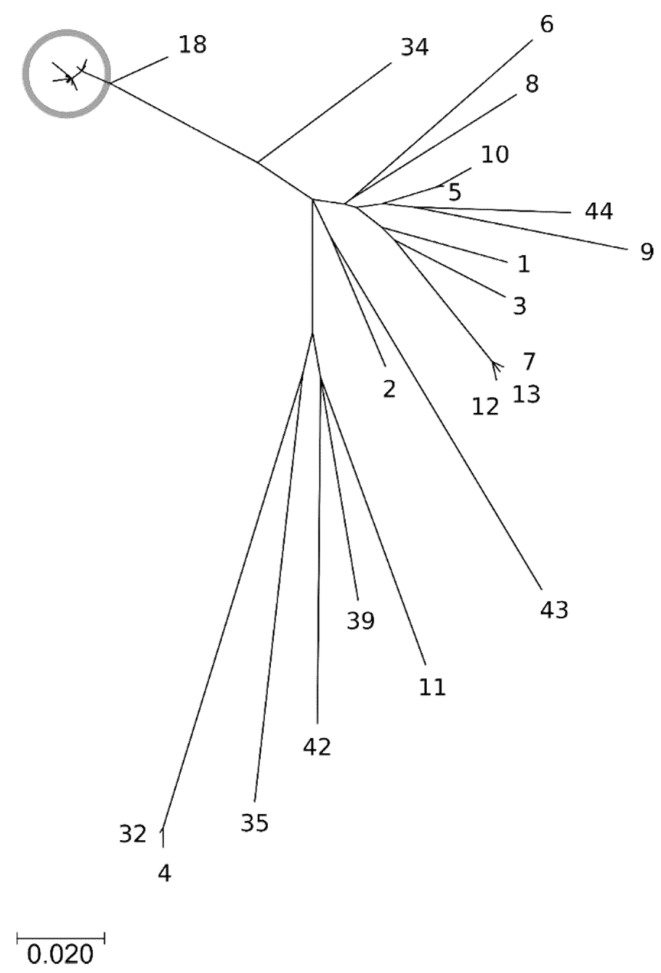

B 19

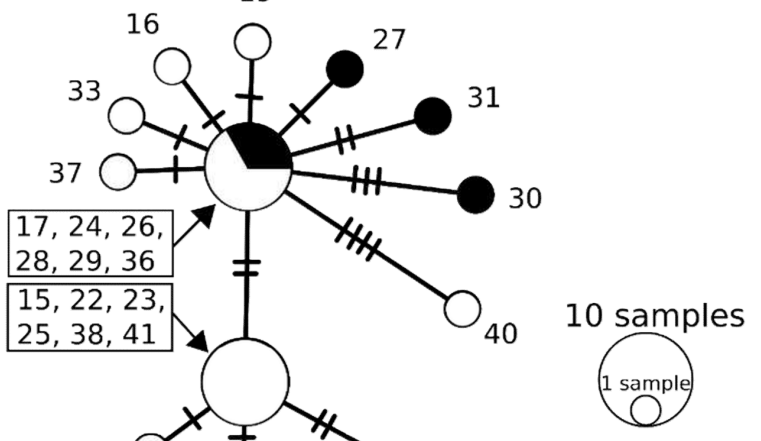

Epidemiological link A family outbreak Ounknown

FIGURE 3 | The phylogenetic analysis results obtained using EHEC 0121 whole genome sequences. The isolate number denotes the serial number in Table 1. (A) Maximum likelihood tree. The scale bar shows the number of substitutions per site. The gray circle indicates the clustered isolates. (B) Median joining network generated from SNPs of the clustered isolates, which are shown in the gray circle in (A). The sizes and colors of the circles represent the number of isolates and the epidemiological link between the isolates, respectively. A crossing bar on a connecting line shows one SNP locus.

length $=216.0 \mathrm{bp}$ ) were not mapped. After de novo assembly of the unmapped reads and subsequent annotation, 42 contigs (>300 bp) were generated. Most of the contigs were derived from prophages or transposon sequences. Excluding these mobile genetic elements, we designed primer pairs to detect two coding sequence (CDS) regions that were specific to isolate 14. Both CDSs encoded hypothetical proteins that were highly unique to the clustered isolates. Two pairs of primers successfully generated a band of the expected size in the clustered isolates (Figure 2). In addition to these isolates, specific amplification was detected in isolate 18 , which was closely related to the clustered isolates. No specific amplification was detected in the other O121 isolates or the isolates of the other serogroups.

\section{DISCUSSION}

Our study showed that molecular typing using WGS was useful for the outbreak investigation of EHEC isolates with high PFGE similarity. Regarding EHEC, O121 isolates often show higher PFGE similarity than other serogroups. Using national surveillance data of PFGE analyses over the last 5 years, O121 has shown the highest mean pairwise similarity in the following major O serogroups: O121, 90.3\%; O157, 84.5\%; O26, 86.6\%; O111, 89.2\%; O103, 76.8\%; and O145, 81.9\% (data not shown). This high PFGE similarity prompted us to use
WGS data to subtype the isolates. Using WGS-based SNPs, we found a plausible epidemiological link between epidemiologically unlinked isolates. In this study, although further tracing of the causative agents or the source could not be conducted, the nationwide spread of highly clonal isolates (Figure 4) suggested that a widely distributed food could be contaminated with EHEC O121. The mean pairwise SNP distance among the isolates in the cluster (2.6) was smaller than the maximum SNP distance in a family outbreak (5) (Figures 2, 3B). This finding also indicates that the isolates in the cluster are genetically indistinguishable. Previous studies of WGS typing from an outbreak investigation showed that EHEC isolates with a known epidemiological link had a distance of less than eight SNPs (Underwood et al., 2013; Joensen et al., 2014; Holmes et al., 2015). The pairwise SNP distances among the cluster in this study ranged from zero to eight and were concordant with previous studies, which suggested a plausible nationwide outbreak. However, interpretation of the pairwise SNP distances requires careful attention. The SNP distance cannot be simply compared because sequencing technology and analytical procedures can vary between studies. Additionally, the core genome size is affected by the sample size and the genetic heterogeneity of the samples. Therefore, the SNP distance should be interpreted with epidemiological information to detect the disease outbreak.

Although the method demonstrated high resolution, WGS-typing is in the trial implementation phase in many 


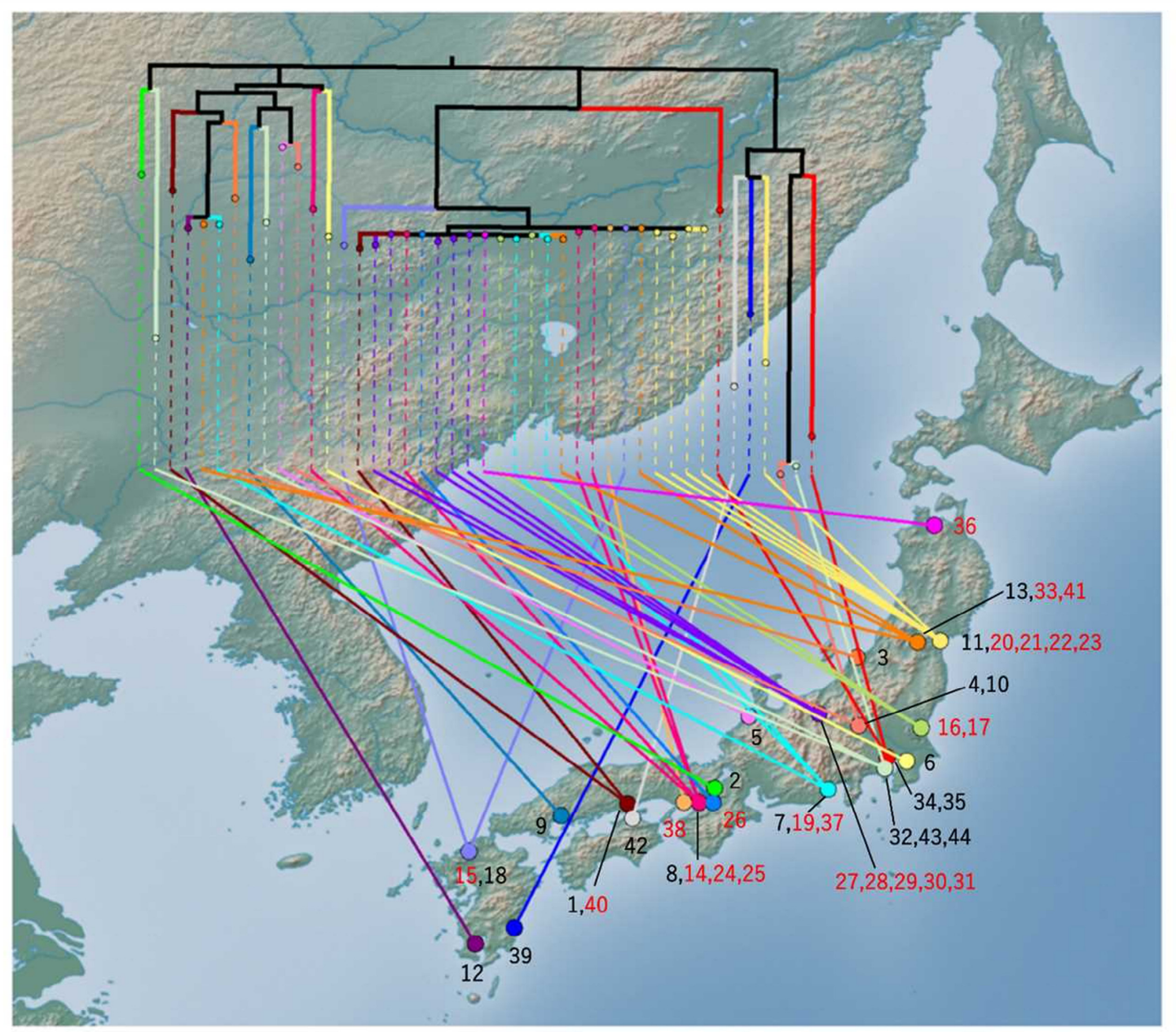

FIGURE 4 | Phylogenetic tree mapped onto the national map of Japan using GenGIS software. The maximum likelihood tree was linked to the isolation site. Black branches in the tree indicate internal edges, which cover multiple prefectures. The isolation sites were mapped to the capital of each prefecture. The number represents the serial number of the isolate. The number of clustered isolates was indicated as red character.

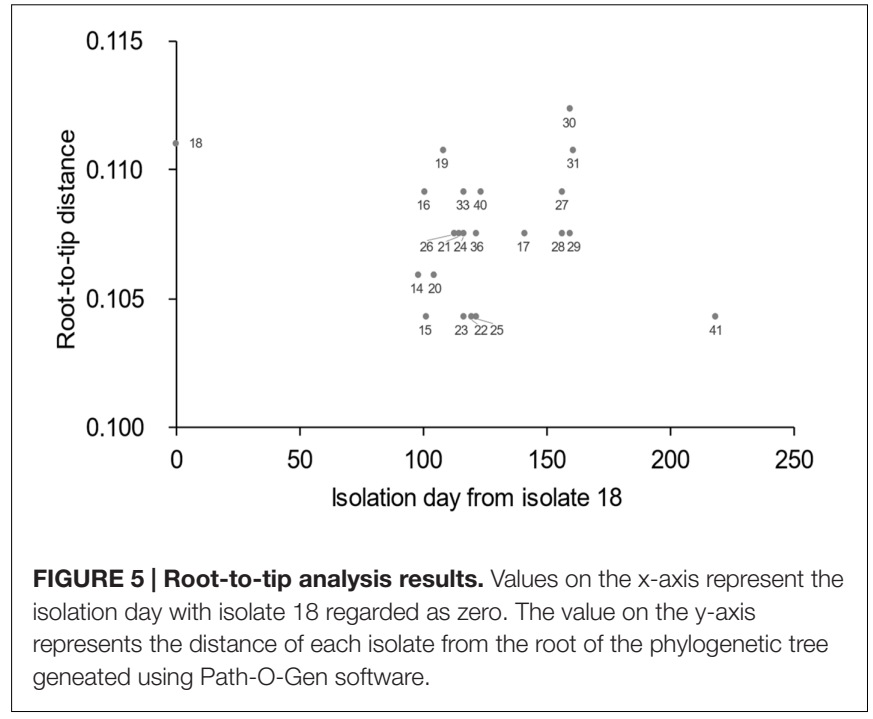

public health laboratories. To date, major implementation of WGS-typing is a complement to conventional methods. When the results obtained through WGS typing and conventional methods are compared, the differences in methodology should be considered. In our results, isolates with no SNPs showed several PFGE patterns. A difference in the PFGE pattern in the same SNP cluster can be explained by an insertion, deletion, and inversion (Iguchi et al., 2006), amplification of a genomic island (Lee et al., 2015), and the gain and loss of mobile genetic elements, such as plasmids, prophages and transposons. Indeed, in silico plasmid replicon typing showed that variation in the same cluster. Inc $\mathrm{B} / \mathrm{O} / \mathrm{K} / \mathrm{Z}$ and FIB plasmids, which are carried by most of the clustered isolates, were not detected from the isolates 22, 27-31, and 37 (Figure 1). These differences in plasmids could affect the result of PFGE. Other mobile genetic elements and a large-scale change in genomic structure could not be detected from our short-read data. Long read data may further improve the discriminatory power. 
In addition to high resolution phylogenetic analyses, WGSs have a wide range of applications, including extracting isolate-specific regions and in silico typing for serotypes, virulence factors and antimicrobial resistance genes (Joensen et al., 2014). In our study, we used WGSs to develop a simple PCR-based detection method for the clustered isolates. Our strategy using the unmapped reads (Figure 1) successfully detected the clustered isolates. Combining WGS-based phylogenetic analyses with an outbreakspecific detection method will be a promising strategy for outbreak investigations, especially outbreaks that are widespread or continue over a prolonged period. This rapid and cost-effective detection method is useful for screening many clinical or food samples to find the link to etiological agents.

\section{CONCLUSION}

The WGS-based phylogenetic analysis revealed the clonal EHEC O121 isolates that could not be identified by conventional PFGE. The results suggest the occurrence of a nationwide outbreak by the clonal isolates. We also developed a WGS-based outbreakspecific detection method. With our strategy, once an outbreak cluster is identified, screening can be conducted by cost-effective PCR. This strategy can be applied to investigations of outbreaks caused by various pathogens.

\section{EHEC WORKING GROUP IN JAPAN}

Hiroko Takenuma (Aomori Prefectural Public Health and Environment Center); Sendai City Institute of Public Health; Junji Seto and Yu Suzuki (Yamagata Prefectural Institute of Public Health); Kyoko Mashiko (Ibaraki Prefectural Institute of Public Health); Shigenori Matsui (Gunma Prefectural Institute of Public Health and Environmental Sciences); Shinichiro Hirai and Eiji Yokoyama (Chiba Prefectural Institute of Public Health); Noriko Konishi, Hiromi Obata, and Akemi Kai (Tokyo Metropolitan Institute of Public Health); Atsuko Ogawa and Yuko Matsumoto (Yokohama City Institute of Health); Ayako Kikuchi (Niigata City Institute of Public Health and Environment); Emiko Kitagawa (Ishikawa Prefectural Institute of Public Health and Environmental Sciences); Hitomi Kasahara

\section{REFERENCES}

Allard, M. W., Strain, E., Melka, D., Bunning, K., Musser, S. M., Brown, E. W., et al. (2016). Practical value of food pathogen traceability through building a wholegenome sequencing network and database. J. Clin. Microbiol. 54, 1975-1983. doi: 10.1128/JCM.00081-16

Bekal, S., Berry, C., Reimer, A. R., Van Domselaar, G., Beaudry, G., Fournier, E., et al. (2016). Usefulness of high-quality core genome singlenucleotide variant analysis for subtyping the highly clonal and the most prevalent Salmonella enterica serovar Heidelberg clone in the context of outbreak investigations. J. Clin. Microbiol. 54, 289-295. doi: 10.1128/JCM. 02200-15

Coil, D., Jospin, G., and Darling, A. E. (2015). A5-miseq: an updated pipeline to assemble microbial genomes from Illumina MiSeq data. Bioinformatics 31, 587-589. doi: 10.1093/bioinformatics/ btu661 and Maki Sekiguchi (Nagano Environmental Conservation Research Institute); Shizuoka Institute of Environmental and Hygiene; Yuji Tsuchiya (Hamamatsu City Health Environment Research Center); Hiromi Nakamura (Osaka City Institute of Public Health and Environmental Science); Kazuko Seto (Osaka Prefectural Institute of Public Health); Nishinomiya Health Center; Junko Tanabe and Mayumi Tsujimoto (Nara Prefectural Institute of Health); Hisahiro Kawai, Hiroko Dannnoue, Ritsuko Ohata, and Hiroshi Nakajima (Okayama Prefectural Institute for Environmental Science and Public Health); Hiroko Yamada and Kanako Masuda (Health Environment Center, Hiroshima Prefectural Technology Research Institute); Kagawa Prefectural Research Institute for Environmental Sciences and Public Health; Fuyuki Okamoto (Fukuoka Institute of Health and Environmental Sciences); Fukuoka City Institute for Hygiene and the Environment; Shuji Yoshino (Miyazaki Prefectural Institute for Public Health and Environment); Kazuyoshi Hozumi (Kagoshima Prefectural Institute for Environmental Research and Public Health).

\section{AUTHOR CONTRIBUTIONS}

KL, TM-I, SI, and MO performed the experiments and wrote the paper. KL, YO, TH, TS, and MK analyzed the data. EHEC Working Group collected the isolates.

\section{FUNDING}

This research is partially supported by the Research Program on Emerging and Re-emerging Infectious Diseases from the Japan Agency for Medical Research and Development (AMED).

\section{ACKNOWLEDGMENTS}

We are grateful to Saomi Ozawa and Yu Takizawa for technical assistance for sequencing and the subsequent analyses. We are also grateful to the members of the EHEC Working Group in Japan for sharing the O121 isolates.

Eyre, D. W., Cule, M. L., Wilson, D. J., Griffiths, D., Vaughan, A., O'connor, L., et al. (2013). Diverse sources of $C$. difficile infection identified on wholegenome sequencing. N. Engl. J. Med. 369, 1195-1205. doi: 10.1056/NEJMoa 1216064

Grad, Y. H., Lipsitch, M., Feldgarden, M., Arachchi, H. M., Cerqueira, G. C., Fitzgerald, M., et al. (2012). Genomic epidemiology of the Escherichia coli O104:H4 outbreaks in Europe, 2011. Proc. Natl. Acad. Sci. U.S.A. 109, 3065-3070. doi: 10.1073/pnas.1121491109

Harris, S. R., Cartwright, E. J., Torok, M. E., Holden, M. T., Brown, N. M., OgilvyStuart, A. L., et al. (2013). Whole-genome sequencing for analysis of an outbreak of meticillin-resistant Staphylococcus aureus: a descriptive study. Lancet Infect. Dis. 13, 130-136. doi: 10.1016/S1473-3099(12)70268-2

Holmes, A., Allison, L., Ward, M., Dallman, T. J., Clark, R., Fawkes, A., et al. (2015). Utility of whole-genome sequencing of Escherichia coli O157 for outbreak detection and epidemiological surveillance. J. Clin. Microbiol. 53, 3565-3573. doi: 10.1128/JCM.01066-15 
Iguchi, A., Iyoda, S., Terajima, J., Watanabe, H., and Osawa, R. (2006). Spontaneous recombination between homologous prophage regions causes large-scale inversions within the Escherichia coli O157:H7 chromosome. Gene 372, 199-207. doi: 10.1016/j.gene.2006.01.005

Infectious Agents Surveillance Report (2016). Enterohemorrhagic Escherichia coli infection, as of April 2016, Japan [Online]. Available: http://www.nih.go.jp/niid/ en/iasr-vol37-e/865-iasr/6488-435te.html [accessed August 2, 2016]

Izumiya, H., Pei, Y. X., Terajima, J., Ohnishi, M., Hayashi, T., Iyoda, S., et al. (2010). New system for multilocus variable-number tandem-repeat analysis of the enterohemorrhagic Escherichia coli strains belonging to three major serogroups: O157, O26, and O111. Microbiol. Immunol. 54, 569-577. doi: 10.1111/j.13480421.2010.00252.x

Joensen, K. G., Scheutz, F., Lund, O., Hasman, H., Kaas, R. S., Nielsen, E. M., et al. (2014). Real-time whole-genome sequencing for routine typing, surveillance, and outbreak detection of verotoxigenic Escherichia coli. J. Clin. Microbiol. 52, 1501-1510. doi: 10.1128/JCM.03617-13

Kumar, S., Stecher, G., and Tamura, K. (2016). MEGA7: molecular evolutionary genetics analysis version 7.0 for bigger datasets. Mol. Biol. Evol. 33, 1870-1874. doi: 10.1093/molbev/msw054

Kurtz, S., Phillippy, A., Delcher, A. L., Smoot, M., Shumway, M., Antonescu, C., et al. (2004). Versatile and open software for comparing large genomes. Genome Biol. 5:R12. doi: 10.1186/gb-2004-5-2-r12

Lee, K., Kusumoto, M., Sekizuka, T., Kuroda, M., Uchida, I., Iwata, T., et al. (2015). Extensive amplification of GI-VII-6, a multidrug resistance genomic island of Salmonella enterica serovar Typhimurium, increases resistance to extended-spectrum cephalosporins. Front. Microbiol. 6:78. doi: 10.3389/fmicb. 2015.00078

Li, W., Raoult, D., and Fournier, P. E. (2009). Bacterial strain typing in the genomic era. FEMS Microbiol. Rev. 33, 892-916. doi: 10.1111/j.1574-6976.2009. 00182.x

Noller, A. C., Mcellistrem, M. C., Pacheco, A. G., Boxrud, D. J., and Harrison, L. H. (2003). Multilocus variable-number tandem repeat analysis distinguishes outbreak and sporadic Escherichia coli O157:H7 isolates. J. Clin. Microbiol. 41, 5389-5397. doi: 10.1128/JCM.41.12.5389-5397.2003

Parks, D. H., Mankowski, T., Zangooei, S., Porter, M. S., Armanini, D. G., Baird, D. J., et al. (2013). GenGIS 2: geospatial analysis of traditional and genetic biodiversity, with new gradient algorithms and an extensible plugin framework. PLoS ONE 8:e69885. doi: 10.1371/journal.pone.0069885

Pei, Y., Terajima, J., Saito, Y., Suzuki, R., Takai, N., Izumiya, H., et al. (2008). Molecular characterization of enterohemorrhagic Escherichia coli O157:H7 isolates dispersed across Japan by pulsed-field gel electrophoresis and multiple-locus variable-number tandem repeat analysis. Jpn. J. Infect. Dis. 61, 58-64.

Pennington, H. (2010). Escherichia coli O157. Lancet 376, 1428-1435. doi: 10.1016/ S0140-6736(10)60963-4
Rambaut, A., Lam, T. T., Max Carvalho, L., and Pybus, O. G. (2016). Exploring the temporal structure of heterochronous sequences using TempEst (formerly Path-O-Gen). Virus Evol. 2:vew007. doi: 10.1093/ve/vew007

Salipante, S. J., Sengupta, D. J., Cummings, L. A., Land, T. A., Hoogestraat, D. R., and Cookson, B. T. (2015). Application of whole-genome sequencing for bacterial strain typing in molecular epidemiology. J. Clin. Microbiol. 53, 1072-1079. doi: 10.1128/JCM.03385- 14

Scheutz, F., Teel, L. D., Beutin, L., Pierard, D., Buvens, G., Karch, H., et al. (2012). Multicenter evaluation of a sequence-based protocol for subtyping Shiga toxins and standardizing Stx nomenclature. J. Clin. Microbiol. 50, 2951-2963. doi: 10.1128/JCM.00860-12

Sekizuka, T., Yamashita, A., Murase, Y., Iwamoto, T., Mitarai, S., Kato, S., et al. (2015). TGS-TB: total genotyping solution for Mycobacterium tuberculosis using short-read whole-genome sequencing. PLoS ONE 10:e0142951. doi: 10. 1371/journal.pone.0142951

Tamura, K., and Nei, M. (1993). Estimation of the number of nucleotide substitutions in the control region of mitochondrial DNA in humans and chimpanzees. Mol. Biol. Evol. 10, 512-526.

Underwood, A. P., Dallman, T., Thomson, N. R., Williams, M., Harker, K., Perry, N., et al. (2013). Public health value of next-generation DNA sequencing of enterohemorrhagic Escherichia coli isolates from an outbreak. J. Clin. Microbiol. 51, 232-237. doi: 10.1128/JCM.01696-12

Wirth, T., Falush, D., Lan, R. T., Colles, F., Mensa, P., Wieler, L. H., et al. (2006). Sex and virulence in Escherichia coli: an evolutionary perspective. Mol. Microbiol. 60, 1136-1151. doi: 10.1111/j.1365-2958.2006.05172.x

Zhou, Z., Mccann, A., Weill, F. X., Blin, C., Nair, S., Wain, J., et al. (2014). Transient Darwinian selection in Salmonella enterica serovar Paratyphi A during 450 years of global spread of enteric fever. Proc. Natl. Acad. Sci. U.S.A. 111, 12199-12204. doi: 10.1073/pnas.1411012111

Conflict of Interest Statement: The authors declare that the research was conducted in the absence of any commercial or financial relationships that could be construed as a potential conflict of interest.

The reviewer AMG and handling Editor declared their shared affiliation, and the handling Editor states that the process nevertheless met the standards of a fair and objective review.

Copyright (C) 2017 Lee, Morita-Ishihara, Iyoda, Ogura, Hayashi, Sekizuka, Kuroda, Ohnishi and EHEC Working Group in Japan. This is an open-access article distributed under the terms of the Creative Commons Attribution License (CC BY). The use, distribution or reproduction in other forums is permitted, provided the original author(s) or licensor are credited and that the original publication in this journal is cited, in accordance with accepted academic practice. No use, distribution or reproduction is permitted which does not comply with these terms. 